\title{
Experiências Obtidas com o Programa Ensinar com Pesquisa da Pró-Reitoria de Graduação da USP na Área de Eficiência Energética
}

\author{
Fernando de Lima Caneppele, José Antonio Rabi \\ Departamento de Engenharia de Biossistemas, Faculdade de Zootecnia e Engenharia de Alimentos da \\ Universidade de São Paulo
}

* Autores para correspondência: caneppele@usp.br; jrabi@usp.br

\section{RESUMO}

Na universidade a docência e a aprendizagem só serão significativas se forem sustentadas por uma permanente atividade de construção do conhecimento. $\mathrm{O}$ aluno consegue aprender significativamente se tal processo se der como construção do conhecimento. Incorporar temas relacionados à eficiência energética nos currículos dos cursos de graduação é importante por fatores como as necessidades do mercado de trabalho e também pela criação de uma cultura de combate ao desperdício dos recursos energéticos. O presente relato mostra alguns dos resultados do projeto Aplicação dos Conceitos de Auditoria Energética no Campus da USP de Pirassununga, apoiado pelo Programa Ensinar com Pesquisa nos editais 2014 e 2015. A participação do aluno nesse projeto (e nessa área temática) teve como objetivo vivenciar na prática, bem como aprofundar em campo, conceitos desenvolvidos nas disciplinas relacionadas à energia elétrica e à eficiência energética dos cursos de Engenharia de Biossistemas e de Engenharia de Alimentos oferecidos na FZEA-USP. A meta supracitada foi atingida, visto que os discentes bolsistas aprofundaram seus conhecimentos na área de eficiência energética, ao passo que os alunos de graduação desfrutaram de aulas mais atrativas sobre esse tema, uma vez que os conceitos desenvolvidos faziam parte do seu cotidiano.

Palavras-Chave: Ensino-Aprendizagem; Conservação de Energia; Ensino-Vivência.

\begin{abstract}
In the university teaching and learning will be meaningful only if they are supported by a permanent knowledge construction activity. Students can significantly learn through knowledge construction rationale. Incorporating energy efficiency issues in undergraduate curricula is important in view of not only labor market needs but also creating a culture of mitigating energy waste. This report shows some results from the project Implementation of Energy Audit Concepts at USP Campus in Pirassununga supported by Teaching with Research programs related to 2014 and 2015 calls. Student participation in the project (and in this thematic area) aimed at hands-on practicing while grasping in loco concepts related to energy and energy efficiency as developed throughout courses offered in either Biosystems or Food Engineering programs at FZEA-USP. Aforesaid project goal was successfully accomplished inasmuch as mentee (i.e. project) students have indeed deepened their knowledge about energy efficiency while undergraduate students have profited from more attractive classes on this subject as concepts became closer to their daily lives.
\end{abstract}

Keywords: Teaching-Learning; Energy Conservation; Hands-On Learning.

\section{Introdução}

Severino (2009) defende que, na universidade, a docência e a aprendizagem só serão significativas se forem sustentadas por uma permanente atividade de construção do conhecimento. O professor precisa da prática da pesquisa para ensinar eficazmente; o aluno necessita dela para aprender eficaz e significativamente; a comunidade precisa da pesquisa para dispor de produtos do conhecimento; e a Universidade, para ser mediadora da educação. O aluno só consegue aprender significativamente se sua aprendizagem se der como construção do conhecimento. 
O mesmo autor ressalta que ensino, pesquisa e extensão universitários efetivamente se articulam, mas a partir da pesquisa. Em outras palavras, somente se aprende e se ensina pesquisando; somente se prestam serviços à comunidade se tais serviços nascerem e se nutrirem da pesquisa. Tem-se uma sistematização de acordo com a qual educar (ensinar e aprender) significa conhecer; por sua vez, conhecer significa construir o objeto; por fim, construir o objeto significa pesquisar. Assim, a aprendizagem, a docência e o ensino na universidade apenas serão significativos se forem sustentados por uma permanente atividade de construção do conhecimento.

De acordo com Pinto et al. (2001), a necessidade de incorporar temas referentes à eficiência energética nos currículos dos cursos de graduação justifica-se (i) pelas necessidades do mercado de trabalho, (ii) pela criação de uma cultura de combate ao desperdício dos recursos energéticos, (iii) pela formação de profissionais integrados ao contexto socioeconômico atual e (iv) pela formação de multiplicadores.

Uma das definições de eficiência energética é que se trata de uma atividade que busca melhorar o uso das fontes de energia. A utilização racional de energia, chamada também simplesmente de eficiência energética, consiste em usar de modo eficiente a energia para se obter um determinado resultado (ABESCO, 2016).

$\mathrm{O}$ combate ao desperdício de energia funciona como uma fonte virtual de produção de energia elétrica, o que quer dizer que a energia não desperdiçada por um consumidor pode ser utilizada por outro. Essa é a fonte de produção de energia mais econômica e mais limpa que existe, pois não agride o meio. Para a disseminação dessa nova cultura, deve-se atuar em dois focos principais, que são: (i) a vertente humana; e (ii) a vertente tecnológica (PINTO et al., 2007).

A aplicação da eficiência energética em diversos setores de nossa sociedade é uma prática que deve ser considerada essencial nos dias atuais. $\mathrm{Na}$ área industrial, a eficientização energética pode trazer significativa redução de custos, bem como aumento no rendimento energético de equipamentos e instalações, com a consequente melhoria da qualidade dos produtos fabricados (COPEL, 2005).

\section{Desenvolvimento}

O projeto Aplicação dos Conceitos de Auditoria Energética no Campus da USP de Pirassununga (apoiado pelo "Programa Ensinar com Pesquisa", editais 2014 e 2015) foi composto de três etapas: (i) revisão bibliográfica; (ii) estudo do local/departamento e realização de coleta de dados elétricos; e (iii) análise dos dados elétricos. Cada uma dessas etapas é abordada a seguir.

\section{(i) Revisão Bibliográfica}

Inicialmente, os alunos bolsistas participantes das duas edições do projeto elaboraram uma revisão bibliográfica para ampliação do domínio e conhecimento do estado da arte do tema eficiência energética, suas implicações e aplicações.

Para realizar esse estudo, foi necessário por parte dos alunos conhecer conceitos relativos à eletricidade tais como: energias ativa, reativa e aparente; potências ativa, reativa e aparente; demandas e cargas; fatores de potência, de demanda e de carga; curvas de carga do sistema; e regimes tarifários. Esses conhecimentos foram obtidos através das aulas e de pesquisas bibliográficas.

\section{(ii) Estudo do Local/Departamento e Realização de Coleta de Dados Elétricos}

O campus Fernando Costa (antigo Pirassununga) é o maior campus da USP em extensão territorial. Trata-se de uma fazenda com 23.333.204,00 $\mathrm{m}^{2}$ de área total, 26.535,55 m de perímetro e $80.594,00$ $\mathrm{m}^{2}$ de área edificada (USP, 2013). Atualmente, o campus engloba as seguintes unidades:

- Prefeitura do campus (PUSP-FG);

- Faculdade de Zootecnia e Engenharia de Alimentos (FZEA);

- Faculdade de Medicina Veterinária e Zootecnia (FMVZ).

Tendo em mente a grande extensão territorial do campus e o grande número de departamentos e seções que compõe cada uma de suas unidades, 
foram escolhidos locais nos quais se concentraram as coletas de dados elétricos e estudos realizados em duas edições anteriores (a saber, 2013 e 2014) deste projeto no âmbito do Programa Ensinar com Pesquisa.

Os dados apresentados neste trabalho se referem ao Bloco Didático do Departamento de Engenharia de Biossistemas. Nesse local foram coletados dados elétricos através da instalação de registradores eletrônicos de sistemas elétricos trifásicos. Coletaram-se os dados de acordo com as definições adotadas na resolução normativa n. 414, de 9 de setembro de 2010, da Agência Nacional de Energia Elétrica (ANEEL), que estabelece as condições gerais de fornecimento de energia elétrica.

Os equipamentos utilizados para coleta de dados foram os analisadores portáteis modelo Saga 4500 cedidos pelo Laboratório de Eficiência Energética e Simulação de Processos da FZEA/USP e o modelo Saga 4000 cedido pelo Laboratório de Energização Rural da Faculdade de Ciências Agronômicas da Unesp de Botucatu. Para a análise dos dados foi utilizado o software da família Plawin fornecido com o kit dos analisadores da Família Saga 4000 e 4500 .

\section{(iii) Análise dos Dados Elétricos}

A seguir são mostradas partes das coletas, tratamento e análises de dados que foram coletados junto à entrada de energia elétrica do bloco didático do Departamento de Engenharia de Biossistemas da USP - campus Fernando Costa. O período total de medição nesse local foi de 8 a 22 de novembro de 2014. A Tabela 1 resume os dados obtidos, enquanto o Anexo mostra os dados para o período de um dia de coleta

A Figura 1 mostra o comportamento da potência ativa trifásica na entrada de energia do bloco didático do Departamento de Engenharia de Biossistemas da USP - campus Fernando Costa.

A Figura 2 mostra o comportamento da potência ativa trifásica em um dia de funcionamento normal do bloco didático do Departamento de Engenharia de Biossistemas da USP - campus Fernando Costa.

\begin{tabular}{|c|c|c|c|c|}
\hline \multirow{2}{*}{ Variáveis } & \multirow{2}{*}{ Tipo (unidade) } & \multicolumn{3}{|c|}{ Valores } \\
\hline & & Médio & Máximo & Mínimo \\
\hline \multirow{3}{*}{ Tensões } & $\mathrm{V} 1(\mathrm{~V})$ & 126,6 & 129,1 & 120,9 \\
\hline & $\mathrm{V} 2(\mathrm{~V})$ & 127,2 & 129,6 & 120,5 \\
\hline & V3 (V) & 127,5 & 129,8 & 120,3 \\
\hline \multirow{3}{*}{ Correntes } & I1 (A) & 21,4 & 162,4 & 4,2 \\
\hline & $\mathrm{I} 2(\mathrm{~A})$ & 21,5 & 164,5 & 2,8 \\
\hline & I3 (A) & 23,1 & 198,0 & 0,0 \\
\hline \multirow{4}{*}{$\begin{array}{c}\text { Potências } \\
\text { Ativas }\end{array}$} & $\mathrm{P} 1(\mathrm{~W})$ & 2473,0 & 18434,0 & 473,0 \\
\hline & P2 (W) & 2564,0 & 20660,0 & 304,0 \\
\hline & P3 (W) & 2674,0 & 23118,0 & 0,0 \\
\hline & $\mathrm{PT}(\mathrm{W})$ & 7711,0 & 60521,0 & 780,0 \\
\hline Fator de Potência & FPT & $0,95(\mathrm{I})$ & $0,99(\mathrm{I}) / 1,00(\mathrm{G}) *$ & $0,85(\mathrm{I}) / 0,92(\mathrm{G}) *$ \\
\hline
\end{tabular}

Tabela 1 - Resumo dos dados elétricos coletados junto à entrada do bloco didático do Departamento de Engenharia de Biossistemas da USP - campus Fernando Costa. 


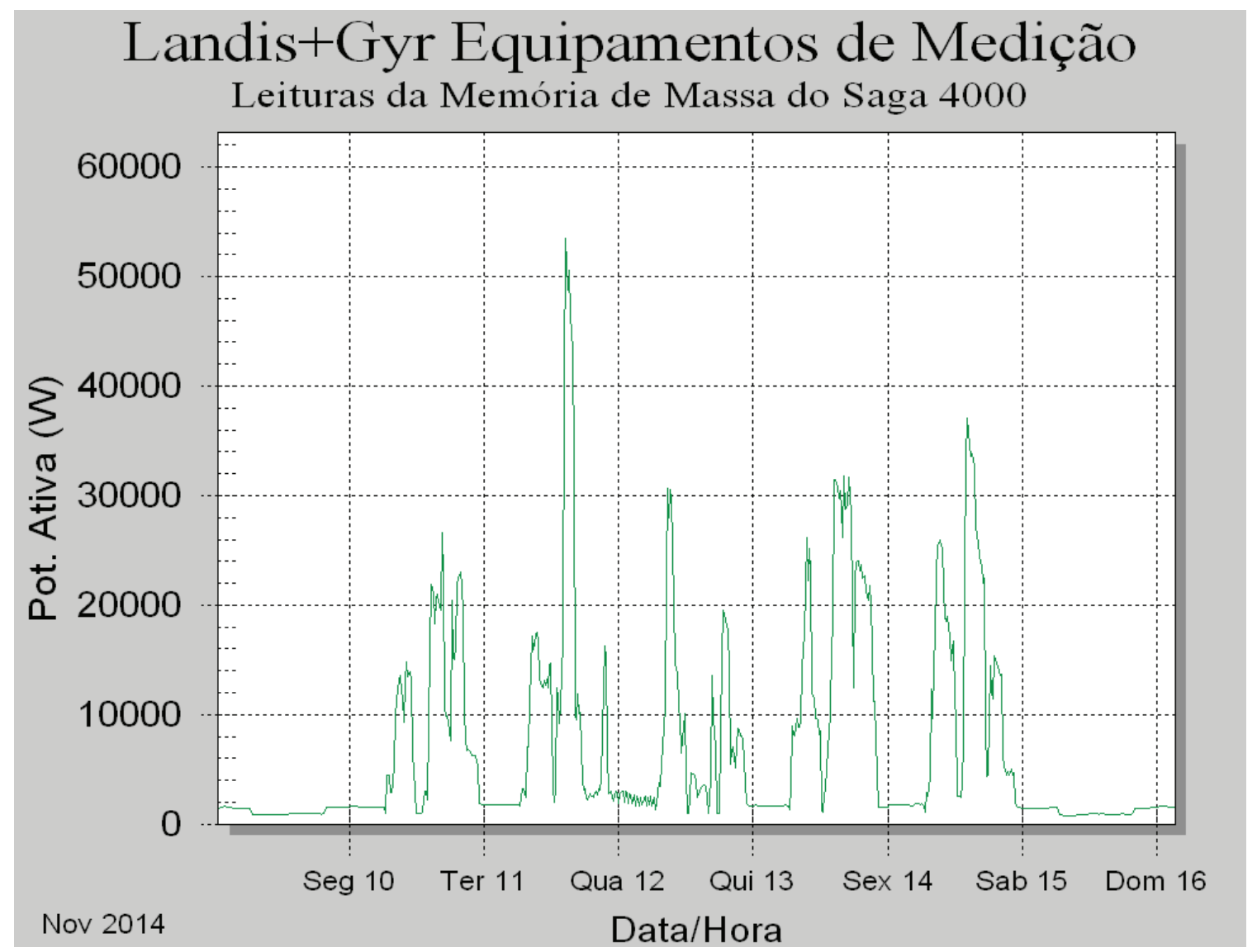

Figura 1 - Potência ativa trifásica junto à entrada do bloco didático do Departamento de Engenharia de Biossistemas da USP - campus Fernando Costa.

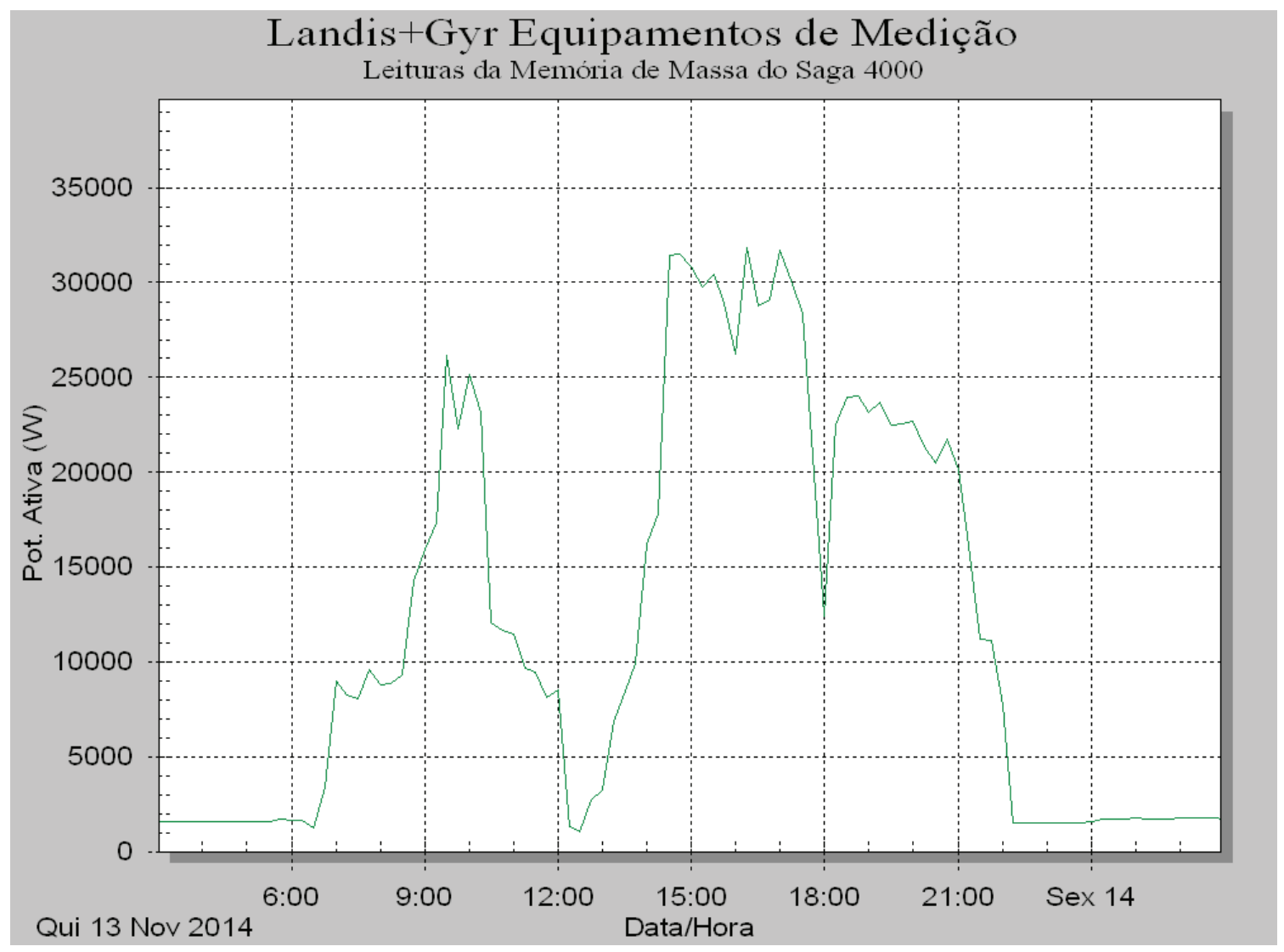

Figura 2 - Potência ativa trifásica - comportamento em dia normal durante a semana junto à entrada do bloco didático do Departamento de Engenharia de Biossistemas da USP - campus Fernando Costa. 
Nota-se que as variações verificadas são próprias do funcionamento do sistema elétrico, ou seja, com picos de consumo nos dias da semana e variações na carga durante o dia.

Os alunos do referido programa participaram de todas as fases práticas do projeto, desde a instalação dos equipamentos de medição, passando pela coleta via computador e análises. Após a coleta dos dados, puderam utilizar o software que acompanha o instrumento e aplicar os conceitos desenvolvidos em sala de aula e em pesquisas teóricas no início de sua participação. Os alunos e docentes envolvidos fizeram diversas análises, algumas das quais são mostradas no presente trabalho.

Nos relatórios finais do projeto, os alunos bolsistas fizeram um modelo de auditoria energética, com revisão bibliográfica, metodologias e resultados mais detalhados.

Os dados e experiências obtidos neste projeto foram incorporados nas aulas das disciplinas relacionadas à eletricidade e à eficiência energética oferecidas nos cursos de graduação em Engenharia de Alimentos e Engenharia de Biossistemas da FZEA-USP.

As situações do cotidiano despertam muito a curiosidade dos alunos. Saber, por exemplo, como é consumida a energia elétrica de um local que eles frequentam diariamente faz com que os alunos possam pensar a respeito disso, atuando também como colaboradores quanto ao uso racional de energia elétrica.

Observou-se nas aulas, após a coleta e discussão dos dados, que os alunos ficaram mais atentos em relação aos conceitos relacionados à energia elétrica e à eficiência energética.

\section{Conclusão}

O projeto Aplicação dos Conceitos de Auditoria Energética no Campus da USP de Pirassununga (apoiado pelo "Programa Ensinar com Pesquisa", editais 2014 e 2015) se propôs a instrumentalizar alunos de graduação em Engenharia de Biossistemas e/ou em Engenharia de Alimentos através de aplicação dos conceitos desenvolvidos nas disciplinas relacionadas à energia elétrica e à eficiência energética.
Compreender como se "consome" a energia é passo fundamental para a implantação de programas de eficiência energética. A verificação de picos de consumo, de data e hora das ocorrências, dos perfis de consumo, por exemplo, proporciona a compreensão do sistema elétrico em estudo e a possibilidade de sucesso quando da implantação de programas de conservação.

Para os alunos bolsistas, houve a oportunidade de aprofundar os conhecimentos obtidos em sala de aula e de colocá-los em prática com ações deste projeto. Para os demais alunos de graduação, as aulas sobre os temas do projeto tornaram-se mais atrativas, uma vez que os conceitos desenvolvidos faziam parte do seu cotidiano.

\section{Agradecimentos}

Os autores agradecem à Pró-Reitoria de Graduação da Universidade de São Paulo e à Comissão de Graduação da Faculdade de Zootecnia e Engenharia de Alimentos (FZEA) da USP, campus Fernando Costa (em Pirassununga), a participação no Programa Ensinar com Pesquisa da USP.

Os autores também agradecem ao Laboratório de Eficiência Energética e Simulação de Processos da FZEA/USP, sob a responsabilidade do professor Celso Eduardo Lins de Oliveira, e ao Laboratório de Energização Rural da Faculdade de Ciências Agronômicas da Unesp de Botucatu, sob a responsabilidade do professor Odivaldo José Seraphim, pela cessão de equipamentos utilizados neste projeto.

\section{Referências Bibliográficas}

ABESCO - Associação Brasileira das Empresas de Serviços de Conservação de Energia. "O Que É Eficiência Energética". Disponível em: <www.abesco. com.br/pt/o-que-e-eficiencia-energetica-ee $>$. Acessado em 2 set. 2016.

COPEL - Companhia Paranaense de Energia. $M a$ nual de Eficiência Energética na Indústria. Curitiba, 2005. $139 \mathrm{p}$.

PINTO, D. P.; OLIVEIRA, E. J. \& BRAGA, H. A. C. "A Disciplina de Eficiência Energética do Curso de Engenharia Elétrica da UFJF". Anais do XXIX Congresso Brasileiro de Ensino de Engenharia. Porto Alegre: Cobenge, 2001. CD-ROM.

; BRAGA, H. A. C. \& SILVA JUNIOR, J. 
P. "A Disciplina Eficiência Energética: Características e Metodologia de Ensino-Aprendizagem". Revista de Ensino de Engenharia, Brasília, vol. 26, n. 1, pp. 43-51, 2007.

SEVERINO, A. J. "Docência Universitária: a Pes- quisa como Princípio Pedagógico". Revista @mbienteeducaşão, São Paulo, vol. 2, n. 1, pp. 120-128, jan.jul. 2009.

USP - Universidade de São Paulo. Anuário Estatístico USP. São Paulo: VREA/USP, 2013. 437 p.

\section{Anexo}

$\mathrm{Na}$ tabela a seguir são apresentadas 47 coletas automáticas. Listam-se, a título de exemplo, apenas dez dos 28 canais de dados do analisador utilizado no experimento.

\begin{tabular}{|c|c|c|c|c|c|c|c|c|c|c|c|c|}
\hline \multicolumn{13}{|c|}{ Saga 4000 - Landis + Gyr } \\
\hline & & & $\begin{array}{c}\text { Canal } \\
03\end{array}$ & $\begin{array}{c}\text { Canal } \\
06\end{array}$ & $\begin{array}{c}\text { Canal } \\
09\end{array}$ & $\begin{array}{c}\text { Canal } \\
11\end{array}$ & $\begin{array}{c}\text { Canal } \\
12\end{array}$ & $\begin{array}{c}\text { Canal } \\
13\end{array}$ & $\begin{array}{c}\text { Canal } \\
17\end{array}$ & $\begin{array}{c}\text { Canal } \\
25\end{array}$ & $\begin{array}{c}\text { Canal } \\
29\end{array}$ & $\begin{array}{c}\text { Canal } \\
33\end{array}$ \\
\hline Registro & Data & Hora & $\mathrm{V} 1$ & $\mathrm{~V} 2$ & $\mathrm{~V} 3$ & I1 & $\mathrm{I} 2$ & $\mathrm{I} 3$ & $\mathrm{Pt}$ & Qcht & $\mathrm{FPt}$ & St \\
\hline & & & $(\mathrm{V})$ & $(\mathrm{V})$ & $(\mathrm{V})$ & (A) & (A) & (A) & $(\mathrm{W})$ & (var) & & \\
\hline 446 & $13 / 11 / 2014$ & 00:00:00 & 127 & 128 & 128 & 7 & 5 & 3 & 1701 & -452 & $-0,97$ & 1760 \\
\hline 448 & $13 / 11 / 2014$ & 00:30:00 & 127 & 127 & 127 & 7 & 5 & 3 & 1754 & -456 & $-0,97$ & 1812 \\
\hline 450 & $13 / 11 / 2014$ & 01:00:00 & 128 & 128 & 128 & 7 & 5 & 3 & 1710 & -437 & $-0,97$ & 1765 \\
\hline 452 & $13 / 11 / 2014$ & 01:30:00 & 127 & 128 & 128 & 7 & 4 & 3 & 1627 & -500 & $-0,96$ & 1702 \\
\hline 454 & $13 / 11 / 2014$ & 02:00:00 & 128 & 129 & 129 & 7 & 4 & 3 & 1635 & -489 & $-0,96$ & 1707 \\
\hline 456 & $13 / 11 / 2014$ & 02:30:00 & 128 & 129 & 129 & 7 & 4 & 3 & 1637 & -488 & $-0,96$ & 1708 \\
\hline 458 & $13 / 11 / 2014$ & 03:00:00 & 128 & 129 & 129 & 7 & 4 & 3 & 1637 & -483 & $-0,96$ & 1706 \\
\hline 460 & $13 / 11 / 2014$ & 03:30:00 & 128 & 129 & 129 & 7 & 4 & 3 & 1635 & -482 & $-0,96$ & 1705 \\
\hline 462 & $13 / 11 / 2014$ & 04:00:00 & 129 & 129 & 129 & 7 & 4 & 3 & 1640 & -476 & $-0,96$ & 1707 \\
\hline 464 & $13 / 11 / 2014$ & 04:30:00 & 129 & 129 & 129 & 7 & 4 & 3 & 1636 & -477 & $-0,96$ & 1704 \\
\hline 468 & $13 / 11 / 2014$ & 05:30:00 & 128 & 129 & 129 & 7 & 4 & 3 & 1623 & -485 & $-0,96$ & 1694 \\
\hline 470 & $13 / 11 / 2014$ & 06:00:00 & 128 & 128 & 129 & 7 & 5 & 3 & 1697 & -430 & $-0,97$ & 1751 \\
\hline 472 & $13 / 11 / 2014$ & 06:30:00 & 128 & 128 & 128 & 6 & 4 & 1 & 1283 & -251 & $-0,98$ & 1307 \\
\hline 474 & $13 / 11 / 2014$ & 07:00:00 & 127 & 128 & 128 & 25 & 25 & 24 & 9000 & 2798 & 0,95 & 9425 \\
\hline 476 & $13 / 11 / 2014$ & 07:30:00 & 126 & 126 & 127 & 23 & 23 & 22 & 8082 & 2680 & 0,95 & 8515 \\
\hline 478 & $13 / 11 / 2014$ & 08:00:00 & 126 & 127 & 127 & 26 & 24 & 24 & 8803 & 3084 & 0,94 & 9328 \\
\hline 480 & $13 / 11 / 2014$ & 08:30:00 & 126 & 127 & 127 & 25 & 26 & 26 & 9355 & 2915 & 0,95 & 9799 \\
\hline 482 & $13 / 11 / 2014$ & 09:00:00 & 125 & 126 & 126 & 46 & 45 & 48 & 15947 & 7287 & 0,91 & 17533 \\
\hline 484 & $13 / 11 / 2014$ & 09:30:00 & 125 & 126 & 126 & 77 & 72 & 78 & 26166 & 11253 & 0,92 & 28484 \\
\hline 486 & $13 / 11 / 2014$ & 10:00:00 & 125 & 126 & 126 & 73 & 64 & 77 & 25185 & 8969 & 0,94 & 26734 \\
\hline 488 & $13 / 11 / 2014$ & 10:30:00 & 125 & 125 & 126 & 33 & 30 & 38 & 12103 & 3791 & 0,95 & 12683 \\
\hline 490 & $13 / 11 / 2014$ & 11:00:00 & 125 & 126 & 126 & 32 & 30 & 37 & 11511 & 4248 & 0,94 & 12270 \\
\hline 492 & $13 / 11 / 2014$ & 11:30:00 & 126 & 126 & 127 & 28 & 24 & 28 & 9483 & 3381 & 0,94 & 10068 \\
\hline 494 & $13 / 11 / 2014$ & 12:00:00 & 127 & 127 & 128 & 25 & 22 & 25 & 8566 & 3033 & 0,94 & 9087 \\
\hline 496 & $13 / 11 / 2014$ & 12:30:00 & 127 & 128 & 128 & 5 & 4 & 0 & 1045 & -102 & $-1,00$ & 1050 \\
\hline 498 & $13 / 11 / 2014$ & 13:00:00 & 126 & 127 & 127 & 9 & 8 & 8 & 3242 & -54 & $-1,00$ & 3243 \\
\hline 500 & $13 / 11 / 2014$ & $13: 30: 00$ & 126 & 127 & 127 & 24 & 27 & 21 & 8472 & 2990 & 0,94 & 8984 \\
\hline 502 & $13 / 11 / 2014$ & 14:00:00 & 125 & 126 & 126 & 54 & 34 & 50 & 16235 & 4138 & 0,97 & 16754 \\
\hline 504 & $13 / 11 / 2014$ & $14: 30: 00$ & 125 & 126 & 126 & 96 & 74 & 96 & 31460 & 10073 & 0,95 & 33033 \\
\hline 506 & $13 / 11 / 2014$ & 15:00:00 & 125 & 126 & 126 & 94 & 70 & 96 & 30823 & 9725 & 0,95 & 32321 \\
\hline 508 & $13 / 11 / 2014$ & 15:30:00 & 125 & 126 & 126 & 95 & 67 & 96 & 30442 & 9928 & 0,95 & 32020 \\
\hline 510 & $13 / 11 / 2014$ & 16:00:00 & 126 & 127 & 127 & 79 & 63 & 79 & 26218 & 9464 & 0,94 & 27874 \\
\hline 512 & $13 / 11 / 2014$ & $16: 30: 00$ & 125 & 126 & 127 & 93 & 62 & 88 & 28784 & 9270 & 0,95 & 30240 \\
\hline
\end{tabular}




\begin{tabular}{|c|c|c|c|c|c|c|c|c|c|c|c|c|}
\hline 514 & $13 / 11 / 2014$ & $17: 00: 00$ & 126 & 127 & 127 & 104 & 64 & 99 & 31727 & 10010 & 0,95 & 33269 \\
\hline 516 & $13 / 11 / 2014$ & $17: 30: 00$ & 126 & 127 & 127 & 68 & 73 & 96 & 28367 & 6167 & 0,98 & 29030 \\
\hline 518 & $13 / 11 / 2014$ & $18: 00: 00$ & 127 & 127 & 128 & 13 & 48 & 45 & 12413 & -163 & $-1,00$ & 12414 \\
\hline 520 & $13 / 11 / 2014$ & $18: 30: 00$ & 126 & 127 & 127 & 41 & 80 & 83 & 23970 & 6551 & 0,96 & 24849 \\
\hline 522 & $13 / 11 / 2014$ & $19: 00: 00$ & 126 & 127 & 127 & 40 & 76 & 80 & 23167 & 6266 & 0,97 & 24000 \\
\hline 524 & $13 / 11 / 2014$ & $19: 30: 00$ & 126 & 127 & 127 & 39 & 74 & 78 & 22502 & 6322 & 0,96 & 23373 \\
\hline 526 & $13 / 11 / 2014$ & $20: 00: 00$ & 124 & 125 & 125 & 41 & 75 & 78 & 22727 & 5859 & 0,97 & 23470 \\
\hline 528 & $13 / 11 / 2014$ & $20: 30: 00$ & 125 & 125 & 126 & 39 & 66 & 69 & 20459 & 5463 & 0,97 & 21175 \\
\hline 530 & $13 / 11 / 2014$ & $21: 00: 00$ & 125 & 126 & 126 & 38 & 65 & 67 & 20176 & 5562 & 0,96 & 20928 \\
\hline 532 & $13 / 11 / 2014$ & $21: 30: 00$ & 126 & 126 & 127 & 32 & 34 & 33 & 11222 & 5622 & 0,89 & 12551 \\
\hline 534 & $13 / 11 / 2014$ & $22: 00: 00$ & 126 & 126 & 126 & 23 & 23 & 22 & 7712 & 3534 & 0,91 & 8483 \\
\hline 536 & $13 / 11 / 2014$ & $22: 30: 00$ & 126 & 127 & 127 & 7 & 4 & 3 & 1543 & -500 & $-0,95$ & 1622 \\
\hline 538 & $13 / 11 / 2014$ & $23: 00: 00$ & 127 & 127 & 128 & 7 & 4 & 3 & 1559 & -491 & $-0,95$ & 1634 \\
\hline 540 & $13 / 11 / 2014$ & $23: 30: 00$ & 127 & 128 & 128 & 7 & 4 & 3 & 1566 & -491 & $-0,95$ & 1641 \\
\hline
\end{tabular}

Publicado em 30/06/2017. 